\title{
Left Cancellative and Right Cancellative Elements of Semigroup of Relations
}

\author{
Chaiwat Namnak and Ratsiri Sanguanwong \\ Department of Mathematics, Faculty of Science, Naresuan University, Phitsanulok 65000
}

Keywords: left cancellative element, right cancellative element, semigroup of relations.

Abstract: Let $R(X)$ denote the set of all relations on a set $X$. Then $R(X)$ becomes a semigroup under composition. The purpose of this paper is to describe the element of $R(X)$ which is left cancellative and right cancellative.

\section{Introduction}

An element $a$ of a semigroup $S$ is said to be left cancellative if for all $b, \mathrm{c} \in S, a b=a c$ implies that $b=c$ and right cancellative if for all $b, \mathrm{c} \in S, b a=c a$ implies that $b=c$

For a nonempty set $X$ a subset $a$ of $X \mathrm{x} X$ is called a (binary) relation on. A binary operation on the set $R(X)$ consisting of all relations on is defined by

$$
\alpha \beta=\{(x, y) \in X \times X \mid(x, z) \in \alpha \text { and }(z, y) \in \beta\}
$$

Then $R(X)$ becomes a semigroup under this multiplication. We call $R(X)$ the semigroup of relations on $X$. For $\alpha \in R(X)$, we denote the domain of $a$ by dom $a$ that is,

and the range of $\alpha$ by ran $\alpha$, that is,

$$
\operatorname{dom} \alpha=\{x \in X \mid(x, y) \in \alpha \text { for some } y \in X\}
$$

If $x \in X$, we define

$$
\operatorname{ran} \alpha=\{y \in X \mid(x, y) \in \alpha \text { for some } x \in X\}
$$

$$
\begin{gathered}
x \alpha=\{y \in X \mid(x, y) \in \alpha\} \text { and } \\
\alpha x=\{y \in X \mid(y, x) \in \alpha\} .
\end{gathered}
$$

As usual, $P(X)$ denote the partial transformation on $X$, the set of all mappings $\alpha$ whose dom $\alpha$ and ran $\alpha$ are subsets of $X$ and $T(X)$ denote the full transformation on $X$, the set of all $\alpha \in P(X)$ with dom $\alpha=X$. Both $P(X)$ and $T(X)$ are subsemigroups of $R(X)$. Our main purpose, we give a necessary and sufficient condition for element of $R(X)$ to be left cancellative and right cancellative.

For the remainder of this paper, we assume that $X$ is a nonempty set and $|X| \geq 2$.

\section{Main Results}

For $\alpha \in R(X)$, denote

$$
\begin{aligned}
X_{\alpha} & =\{x \in X|| x \alpha \mid=1\} \text { and } \\
\alpha_{*} & =\left\{(x, y) \in \alpha \mid x \in X_{\alpha}\right\}
\end{aligned}
$$

The following result is to characterize a left cancellative element of $R(X)$.

Theorem 1.1 Let $\alpha \in R(X)$. Then $a$ is left cancellative if and only if $\operatorname{ran} \alpha_{*}=X$.

Proof Suppose that $\alpha$ is a left cancellative element of $R(X)$. Claim that ran $\alpha=X$, suppose not. Then there exists $x \in X \backslash$ ran $\alpha$. Let $z$ and $w$ be two distinct elements in $X$. Define $\beta, \gamma \in R(X)$ by $\beta=\{(x, w)\}$ and $\gamma=\{(x, z)\}$. Then $\alpha \beta=\varnothing=\alpha \gamma$. Since $\alpha$ is left cancellative, we deduce that $\beta=\gamma$, a contradiction. So we have the claim. If $X_{\alpha}=X$, then $\operatorname{ran} \alpha_{*}=X$. Assume that $X_{\alpha} \neq X$. To show that $\operatorname{ran} \alpha_{*}=X$, suppose not. Then there exists $z \in X \backslash \operatorname{ran} \alpha_{*}$. Since ran $\alpha=X$, there exists $x \in X$, $(x, z) \in \alpha$. Since $z \notin \operatorname{ran} \alpha_{*}$, it follows that $x \notin X_{\alpha}$. Then there exists $w \in X$ such that $w \neq z$ and $(x, w) \in \alpha$. Let $y \in X$ be such that $(y, z) \in \alpha$. Similarly, we obtain that there exists $u \in X$ such that $u \neq z$ and $(y, u) \in \alpha$. Let $\beta, \gamma \in R(X)$ be such that $\beta=X \times\{v\}$ and $\gamma=\beta \backslash\{(z, v)\}$ for some $v \in X$. It is not difficult to verify that $\alpha \beta=\alpha \gamma$. By assumption, we have $\beta=\gamma$ which is a contradiction. Hence $\operatorname{ran} \alpha_{*}=X$. 
Conversely, suppose that ran $\alpha_{*}=X$. Let $\beta, \gamma \in R(X)$ be such that $\alpha \beta=\alpha \gamma$. Let $(x, y) \in \beta$. Since ran $\alpha_{*}=X$, there exists $z \notin X_{\alpha}$ such that $(z, x) \in \alpha$. Thus $(z, y) \in \alpha \beta$ so $(z, y) \in \alpha \gamma$. We then have that $(z, w) \in \alpha$ and $(w, y) \in \gamma$ for some $w \in X$. Since $z \in X_{\alpha}$, we deduce that $x=w$. Thus $(x, y) \in \gamma$, so $\beta \subseteq \gamma$. In a similarly way one can verify that $\gamma \subseteq \beta$. Therefore $\beta=\gamma$ as desired.

The following examples illustrate the applications of Theorem 1.1.

Example 1. Let $\alpha \in R(\mathrm{Z})$ be defined by

$$
\alpha=\{(2 n, n),(2 n+1, n),(2 n+1, n+1) \mid n \in Z\} .
$$

We have that $\alpha_{*}=\{(2 n, n) \mid n \in Z\}$ and $\operatorname{ran} \alpha_{*}=X$. By theorem 1.1, we deduce that $\alpha$ is a left cancellative element of $R(\mathrm{Z})$.

Example 2. Let $\alpha \in R(\mathrm{~N})$ be defined by

$$
\alpha=\{(n, n),(n, n+1) \mid n \in Z\} .
$$

It is easy to see that $\alpha$ is onto, but $\alpha_{*}=\varnothing$. Then $\alpha$ is not a left cancellative element of $R(\mathrm{~N})$.

If $\alpha \in R(X)$ is a mapping, we have $X_{\alpha}=\operatorname{dom} \alpha$ and $\alpha_{*}=\alpha$. The following corollaries are immediately consequence of Theorem 1.1 .

Corollary 1.2 Let $\alpha \in P(X)$. Then $\alpha$ is left cancellative if and only if $\alpha$ is onto.

Corollary 1.3 Let $\alpha \in T(X)$. Then $\alpha$ is left cancellative if and only if $\alpha$ is onto.

For $\alpha \in R(\mathrm{~N})$, denote

$$
\begin{aligned}
X^{\alpha} & =\{y \in X|| \alpha y \mid=1\} \text { and } \\
\alpha^{*} & =\left\{(x, y) \in \alpha \mid y \in X^{\alpha}\right\}
\end{aligned}
$$

Next, to characterize the right cancellative of element of $R(X)$.

Theorem 1.4 Let $\alpha \in R(X)$. Then $\alpha$ is right cancellative if and only if dom $\alpha^{*}=X$.

Proof Suppose that $\alpha$ is a right cancellative element of $R(X)$. Claim that dom $\alpha=X$, suppose not. Then there exists $x \in X \backslash$ dom $\alpha$. Let $z$ and $w$ be two distinct elements in $X$. Define $\beta, \gamma \in R(X)$ by $\beta=\{(w, x)\}$ and $\gamma=\{(z, x)\}$. Then $\beta \alpha=\varnothing=\gamma \alpha$. Since $\alpha$ is left cancellative, we deduce that $\beta=\gamma, \mathrm{a}$ contradiction. Thus we have the claim. If $X^{\alpha}=X$, then $\operatorname{dom} \alpha^{*}=\operatorname{dom} \alpha=X$. Assume that $X^{\alpha} \neq X$. To show that dom $\alpha^{*}=X$, suppose not. Then there exists $z \in X \backslash \operatorname{dom} \alpha^{*}$. Since dom $\alpha=X$, there exists $x \in X,(z, x) \in \alpha$. Since $z \notin \operatorname{dom} \alpha^{*},(z, x) \notin \alpha_{*}$. We then have $x \notin X^{\alpha}$, so there exists $w \in X$ such that $w \neq z$ and $(w, x) \in \alpha$. Let $y \in X$ be such that $(z, y) \in \alpha$. Similarly, we obtain that there exists $u \in X$ such that $u \neq z$ and $(u, y) \in \alpha$. Define $\beta, \gamma \in R(X)$ by $\beta=\{v\} \times X$ and $\gamma=\beta \backslash\{(v, z)\}$ for some $v \in X$. It's easy to verify that $\beta \alpha=\gamma \alpha$. By assumption, we have $\beta=\gamma$ which is a contradiction. Therefore $\operatorname{dom} \alpha^{*}=X$.

Conversely, suppose that dom $\alpha^{*}=X$. Let be such that $\beta, \gamma \in R(X)$. Let $(x, y) \in \beta$. Since dom $\alpha^{*}=X$, there exists $z \in X^{\alpha}$ such that $(y, z) \in \alpha^{*}$. Thus $(x, z) \in \beta \alpha$, so $(x, z) \in \gamma \alpha$. We then have that $(w, z) \in \alpha$ and $(x, w) \in \gamma$ for some $w \in X$. Since $z \in X^{\alpha}$, we deduce that $w=y$. Thus $(x, y) \in \gamma$, so $\beta \subseteq \gamma$. In a similarly way one can verify that $\gamma \subseteq \beta$. Hence $\beta=\gamma$. The proof is now complete.

We now illustrate the use of Theorem 1.4.

Example 3. Let $\alpha \in R(\mathrm{Z})$ be such that

$$
\alpha=\{(n, n+1),(n, 1) \mid n \in Z\}
$$

We then have $\alpha_{*}=\{(n, n+1) \mid n \in Z\}$ and dom $\alpha^{*}=X$ which satisfy Theorem 1.4.

Hence $\alpha$ is a right cancellative element of $R(\mathrm{Z})$.

The following corollaries are clearly obtained from Theorem 1.4.

Corollary 1.5 Let $\alpha \in P(X)$. Then $\alpha$ is right cancellative if and only if $\alpha$ is one-to-one and dom $\alpha=X$.

Corollary 1.6 Let $\alpha \in T(X)$. Then $\alpha$ is right cancellative if and only if $\alpha$ is one-to-one.

For each $\alpha \in R(X)$ we define $\alpha^{-1}$ the inverse of $\alpha$ by

$$
\left.\alpha^{-1}=(x, y) \in X \times X \mid(y, z) \in \alpha\right\} .
$$

It now follows easily that $x \alpha=\alpha^{-1} x$ for all $x \in X$. We have

Corollary 1.7 Let $\alpha \in R(X)$. Then $\alpha$ is left cancellative if and only if $\alpha^{-1}$ is right cancellative. 


\section{References}

[1] Higgins, P. M. 1992. Techniques of Semigroup Theory, New York, Oxford University Press.

[2] Howie, J. M. 1995. Fundamentals of Semigroup Theory, Clarendon Press, Oxford. 